\title{
Impaired Rhodopsin Generation in the Rat Model of Diabetic Retinopathy
}

\author{
Volha V. Malechka, ${ }^{*}$ Gennadiy Moiseyev,${ }^{*}$ Yusuke Takahashi, ${ }^{\dagger}$ Younghwa Shin, ${ }^{*}$ and Jian-xing Ma*
}

From the Departments of Physiology* and Medicine, ${ }^{\dagger}$ Harold Hamm Diabetes Center, University of Oklahoma Health Sciences Center, Oklahoma City, Oklahoma

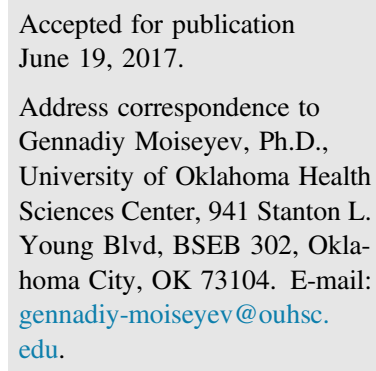

\begin{abstract}
Diabetic retinopathy is a common complication of diabetes mellitus. Diabetic patients experience functional deficits in dark adaptation, contrast sensitivity, and color perception before microvascular pathologies become apparent. Herein, we evaluated early changes in neural retinal function and in retinoid metabolism in the eye in diabetes. Streptozotocin-induced diabetic rats showed decreased aand b-wave amplitudes of scotopic and photopic electroretinography responses 4 months after diabetes induction compared to nondiabetic controls. Although Western blot analysis revealed no difference in opsin expression, rhodopsin content was decreased in diabetic retinas, as shown by a difference in absorbance. Consistently, levels of 11-cis-retinal, the chromophore for visual pigments, were significantly lower in diabetic retinas compared to those in controls, suggesting a retinoid deficiency. Among visual cycle proteins, interphotoreceptor retinoid-binding protein and stimulated by retinoic acid 6 protein showed significantly lower levels in diabetic rats than those in nondiabetic controls. Similarly, serum levels of retinol-binding protein 4 and retinoids were significantly lower in diabetic rats. Overall, these results suggest that retinoid metabolism in the eye is impaired in type 1 diabetes, which leads to deficient generation of visual pigments and neural retinal dysfunction in early diabetes. (Am J Pathol 2017, 187: 2222-2231; http://dx.doi.org/10.1016/j.ajpath.2017.06.007)
\end{abstract}

Diabetic retinopathy (DR) is a common complication of diabetes mellitus ${ }^{1}$ and the leading cause of severe vision loss and blindness in the working-age population in developed countries. ${ }^{2}$ Previous studies of DR were focused on microvascular damage in the retina. ${ }^{3}$ However, recent evidence strongly suggests that DR involves alterations in the neural retina. Patients with diabetes demonstrate functional deficits in dark adaptation, contrast sensitivity, and color perception. ${ }^{4,5}$ These functional changes can be identified before major retinal vascular pathologies, suggesting that they are due to a direct effect of diabetes on the function of the neural retina, rather than secondary to the vascular changes in diabetes. ${ }^{6}$ The molecular basis for these functional changes in early DR is unclear.

The major function of vitamin $\mathrm{A}$ in vision is to serve as a precursor for light-sensitive 11-cis-retinal, the chromophore of visual pigments. In vertebrates, vision begins with the absorption of photon by a light-sensitive visual pigment molecule, which is formed by an apoprotein opsin and a visual chromophore, 11-cis-retinal. ${ }^{7}$ On absorption of light, 11-cis-retinal is isomerized to all-trans-retinal. This isomerization leads to conformational changes of opsin and, thereafter, to phototransduction activation that initiates vision. The regeneration of 11-cis-retinal through a series of reactions between the retinal pigment epithelium (RPE) and the photoreceptor outer segment is referred to as the retinoid or visual cycle (Figure 1).

All-trans-retinal, generated by light, is dissociated from opsin and reduced to all-trans-retinol by all-trans-retinol dehydrogenases in photoreceptors (Figure 1). All-transretinol is then transported from photoreceptors to the RPE. In RPE cells, all-trans-retinol is esterified, forming alltrans-retinyl ester, which is then converted to 11-cis-retinol by RPE65, the isomerase. Generated 11-cis-retinol is

Supported by NIH grants EY018659, EY012231, EY019309, and P20GM104934 (J.-X.M.); Juvenile Diabetes Research Foundation grant 2-SRA-2014-147-Q-R (J.-x.M.); and Oklahoma Center for the Advancement of Science and Technology grant HR13-076 (J.-X.M.).

Disclosures: None declared. 


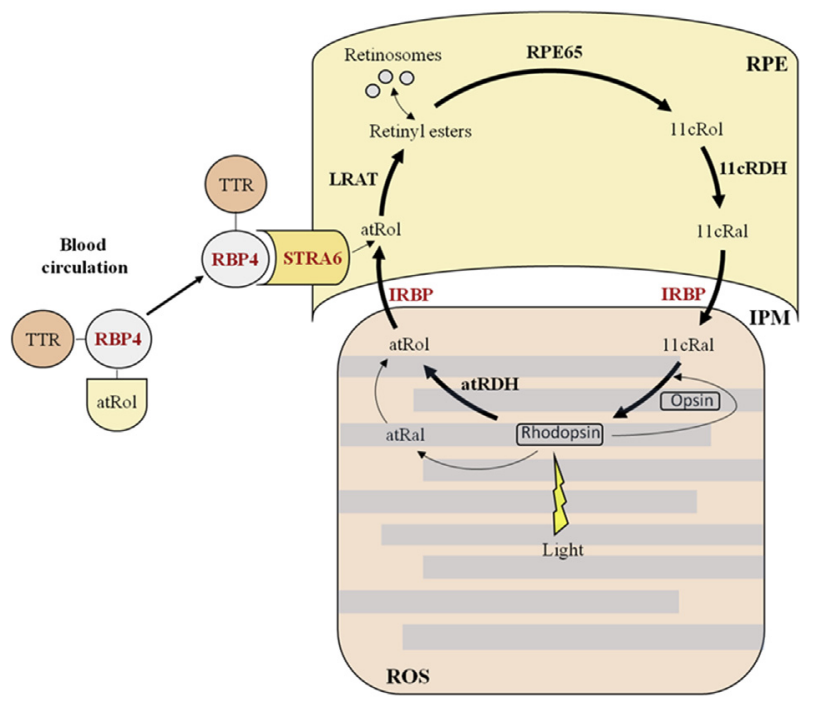

Figure 1 Scheme of retinoid visual cycle. 11cRal, 11-cis-retinal; 11cRDH, 11-cis-retinol dehydrogenase; 11cRol, 11-cis-retinol; atRal, all-trans-retinal; atRDH, all-trans-retinol dehydrogenase; atRol, all-trans-retinol; IPM, interphotoreceptor matrix; IRBP, interphotoreceptor retinoid-binding protein; LRAT, lecithin retinol acyltransferase; RBP4, retinol-binding protein 4; ROS, receptor outer segment; RPE, retinal pigment epithelium; RPE65, retinal pigment epithelium-specific $65-\mathrm{kDa}$ protein; STRA6, stimulated by retinoic acid 6; TTR, transthyretin.

oxidized to 11-cis-retinal by 11-cis-retinol dehydrogenases, including retinol dehydrogenases 5 and 10. Produced 11-cis-retinal is then transported back into photoreceptors. In photoreceptors, 11-cis-retinal binds with opsin to regenerate visual pigments. Uptake of vitamin A into the eye is mediated by stimulated by retinoic acid gene 6 (STRA6), a membrane protein located in the RPE and functioning as a receptor for retinol-binding protein 4 (RBP4). ${ }^{9}$ RBP4 is a carrier of all-trans-retinol (vitamin A) in the circulation. On binding to STRA6, RBP4 releases alltrans-retinol into the RPE, providing retinoids to the visual cycle. ${ }^{10}$ RBP4 delivers retinol from the liver storage to the target cells and tissues, such as the RPE in the eye.

To enter the photoreceptor outer segment, 11-cis-retinal generated in the RPE passes through the interphotoreceptor matrix. Interphotoreceptor retinol-binding protein (IRBP; alias RBP3) is a retinoid-binding protein secreted from photoreceptors and present in the interphotoreceptor space. ${ }^{11}$ It facilitates transport of all-trans-retinol from photoreceptors to the RPE and 11-cis-retinal from the RPE to photoreceptors ${ }^{12}$ (Figure 1).

Visual function deficiencies were observed in patients with defects in genes responsible for the visual cycle. However, the association between retinoid metabolism and DR has not been confirmed. The goal of this study was to investigate the impact of diabetes on the retinoid cycle and metabolism of retinoids in the eye. We found that rhodopsin generation is impaired because of retinoid deficiency in the diabetic eye, which suggests a new pathogenic mechanism for retinal dysfunction in early DR.

\section{Materials and Methods}

\section{Animals}

Male Wistar rats were purchased from Charles River Laboratories (Wilmington, MA). The care and all procedures for the animals were performed in strict agreement with the guidelines for ethical treatment and use of laboratory animals, approved by the Institutional Animal Care and Use Committee of the University of Oklahoma (Oklahoma City).

\section{Induction of Experimental Diabetes}

Diabetes was induced in rats of 10 weeks of age after overnight fasting by a single i.p. injection of freshly prepared streptozotocin (STZ) solution in $0.1 \mathrm{~mol} / \mathrm{L}$ citrate buffer ( $\mathrm{pH} \mathrm{4.5)}$ at the dose of $55 \mathrm{mg} / \mathrm{kg}$ of body weight. Age-matched control rats were injected with the citrate buffer alone. Diabetes was defined if blood glucose levels were continuously $>350 \mathrm{mg} / \mathrm{dL}$, tested by handheld blood glucose meter from the tail vein. The duration of diabetes throughout the study was 4 months.

\section{Retinal Function Test with ERG}

Rats were dark adapted for at least 16 hours and prepared for the full-field electroretinographic (ERG) recording under dim red light. ERG procedures were performed using the Espion $\mathrm{E}^{3}$ system with a Ganzfeld ColorDome (Diagnosys LLC, Lowell, MA). Animals were anesthetized with 100 $\mathrm{mg} / \mathrm{kg}$ of body weight of ketamine (VetOne, Boise, ID) and $10 \mathrm{mg} / \mathrm{kg}$ of body weight of xylazine (AnaSed, Decatur, IL) mixture solution i.p. Pupils were dilated with an application of $1 \%$ Cyclopentolate Hydrochloride Ophthalmic Solution (Alcon Laboratories, Inc., Fort Worth, TX). After observable mydriasis, one drop of Goniovisc (Contacare Ophthalmics and Diagnostics, Gujarat, India) was applied on the cornea of each eye. A ground electrode was placed on a tail, a reference electrode was put in a mouth, and two gold wire loop electrodes were positioned on a corneal surface of the right and left eyes. Rats were placed inside the Ganzfeld sphere. A single flash of $200 \mathrm{~cd} \times \mathrm{s} / \mathrm{m}^{2}$ intensity was applied to induce rod response under fully dark-adapted conditions. Then, animals were exposed to a steady light of $200 \mathrm{~cd} / \mathrm{m}^{2}$ for 2 minutes to obtain cone response to a single flash $\left(600 \mathrm{~cd} \times \mathrm{s} / \mathrm{m}^{2}\right)$ stimulus after rod saturation.

\section{Measurement of Rhodopsin Content}

For determination of the maximal rhodopsin content, rats were dark adapted for at least 16 hours. All procedures were performed under dim red light. Rats were euthanized, and their eyes were removed and their retinas dissected. Immediately after tissue collection, each retina was homogenized in $150 \mu \mathrm{L}$ of $1 \times$ phosphate-buffered saline containing $1 \%(\mathrm{w} / \mathrm{v})$ dodecyl maltoside. Homogenates were 
centrifuged at $70,000 \times g$ for 1 hour. Supernatants were transferred to clean spectrophotometer cuvettes and scanned from 250 to $700 \mathrm{~nm}$ of wavelength using a DU800 spectrophotometer (Beckman Coulter, Inc., Brea, CA). The difference of absorbance spectra between prebleached and post-bleached samples at $500 \mathrm{~nm}$ was used to calculate rhodopsin content using a molar extinction coefficient of $42,000 \mathrm{~mol} / \mathrm{L}^{-1} \mathrm{~cm}^{-1} \cdot{ }^{13}$ Obtained data were normalized to total volume of supernatant and presented as rhodopsin content per retina.

\section{Western Blot Analysis}

For Western blot analysis of lecithin retinol acyltransferase (LRAT), RPE65, IRBP, and STRA6, eyecups of each rat were carefully collected. To measure opsin expression by immunoblotting, retinas were separated from eyecups. Each sample was homogenized separately. Protein concentrations in eyecup homogenates were determined using the Bradford protein assay. ${ }^{14}$ An equal amount of total protein $(30 \mu \mathrm{g})$ from each eyecup sample as well as the same amount of total protein $(50 \mathrm{ng}$ ) from each retina sample was resolved by SDSPAGE and transferred onto a nitrocellulose membrane. The membrane was blocked with 5\% nonfat milk and separately blotted with a 1D4 antibody (1:1000) for opsin as well as an antibody (1:3000) for LRAT (Proteintech, Rosemont, IL), RPE65 (Proteintech), IRBP (Santa Cruz Biotechnology, Dallas, TX), or STRA6 (Abcam, Cambridge, UK). The membrane was then stripped and reblotted with a monoclonal antibody for $\beta$-actin (1:1000). Protein levels of opsin, LRAT, RPE65, IRBP, and STRA6 were semiquantified by densitometry using AlphaView SA software version 3.4.0.0 from ProteinSimple (San Jose, CA). To quantify opsin expression level in diabetic and control retina samples, an opsin monomer band (approximately $40 \mathrm{kDa}$ ) was used for analysis.

\section{LRAT and RPE65 Activity Assays}

LRAT and RPE65 enzymatic activities were measured using high-performance liquid chromatography (HPLC). Rats were euthanized, and their eyes were enucleated. The cornea, lens, and retina were removed, and the remaining eyecup was homogenized and used for LRAT and RPE65 activity assays. Isomerase activity of RPE65 was measured, as described. ${ }^{15}$ Briefly, $50 \mu \mathrm{g}$ of rat RPE homogenate was incubated at $37^{\circ} \mathrm{C}$ for 2 hours in $200 \mu \mathrm{L}$ of reaction buffer $(10 \mathrm{mmol} / \mathrm{L} 1,3$-bis[Tris(hydroxymethyl)-methyl amino] propane ( $\mathrm{pH} 8.0$ ), and $100 \mathrm{mmol} / \mathrm{L} \mathrm{NaCl}$ ) containing $0.2 \mu \mathrm{mol} / \mathrm{L}$ all-trans-retinol, $1 \%(\mathrm{w} / \mathrm{v})$ bovine serum albumin, and $25 \mu \mathrm{mol} / \mathrm{L}$ cellular retinaldehyde-binding protein. The reaction was stopped, and retinoids were extracted with $300 \mu \mathrm{L}$ of methanol and $300 \mu \mathrm{L}$ of hexane and centrifuged at $10,000 \times g$ for 5 minutes. The top layer containing retinoids was collected and analyzed by normal-phase HPLC, as described. ${ }^{15}$ LRAT activity was measured, as described previously. ${ }^{15}$ The reaction mixture was the same as that for the isomerase assay, except for the absence of cellular retinaldehyde-binding protein.

\section{Retinoid Profile Analysis}

For endogenous retinoid analysis in the retina and the RPE, rats were dark adapted for 16 hours and sacrificed under dim red light; their eyes were enucleated. The cornea, lens, and retina were removed, the remaining eyecup was homogenized with a glass grinder in extraction buffer $[10 \mathrm{mmol} / \mathrm{L}$ $\mathrm{NH}_{2} \mathrm{OH}, 50 \%$ ethanol, 50\% 2-( $\mathrm{N}$-morpholino) ethanesulfonic acid, $\mathrm{pH}$ 6.5], and retinoids were extracted with hexane. The solvent was evaporated under argon gas, and dried retinoids were resuspended in $200 \mu \mathrm{L}$ of mobile phase (11.2\% ethyl acetate, $2.0 \%$ dioxane, $1.4 \%$ octanol, $85.4 \%$ hexane) and injected into the HPLC machine (515 HPLC pump; Waters Corp., Milford, MA) for separation using a $5-\mu \mathrm{m}$ sorbent column (Lichosphere SI-60; $4.6 \times 250 \mathrm{~mm}$; Alltech, Deerfield, IL). Each retinoid isomer was quantified from the area of its determined corresponding peak based on synthetic retinoid standards for calibration.

\section{Serum Retinol Measurement}

To measure serum retinol levels, $200 \mu \mathrm{L}$ of rat serum was mixed with $1 \mathrm{~mL}$ of $0.025 \mathrm{~mol} / \mathrm{L} \mathrm{KOH}$ in ethanol. Retinoids were extracted by $10 \mathrm{~mL}$ of hexane, and the solvent was dried out under argon gas. Dried retinoid samples were resuspended in $200 \mu \mathrm{L}$ of mobile phase solvent $(11.2 \%$ ethyl acetate, $2.0 \%$ dioxane, $1.4 \%$ octanol, $85.4 \%$ hexane) and injected into the HPLC machine (515 HPLC pump). All-trans-retinol absorbance was recorded at $320 \mathrm{~nm}$. Serum retinol levels were quantified from the area of the all-transretinol peak using synthetic all-trans-retinol as a standard for calibration (Empower software version 1; Waters Corp.).

\section{Serum RBP4 Measurement}

Rat blood was collected by the cardiac puncture after overnight fasting into serum separator tubes (BD Diagnostics, Franklin Lakes, NJ). After clot formation, blood samples were centrifuged at $2000 \times g$ for 15 minutes at $4^{\circ} \mathrm{C}$. Serum was collected in microfuge tubes. Undiluted serum samples were stored at $-80^{\circ} \mathrm{C}$ until they were analyzed. RBP4 levels in rat serum were measured using a commercial SimpleStep enzyme-linked immunosorbent assay kit (Abcam).

\section{Immunostaining of Eyecup Sections}

Rat eyecups were carefully enucleated and fixed in $4 \%$ paraformaldehyde solution for 2 hours at $4^{\circ} \mathrm{C}$. Then, corneas were punctured in the limbus area, excised, and removed along with the lens. Each eyecup was embedded in OTC compound in a separate mold using liquid nitrogen for rapid freezing. Eyecup samples were cut into sections $(10 \mu \mathrm{m}$ 

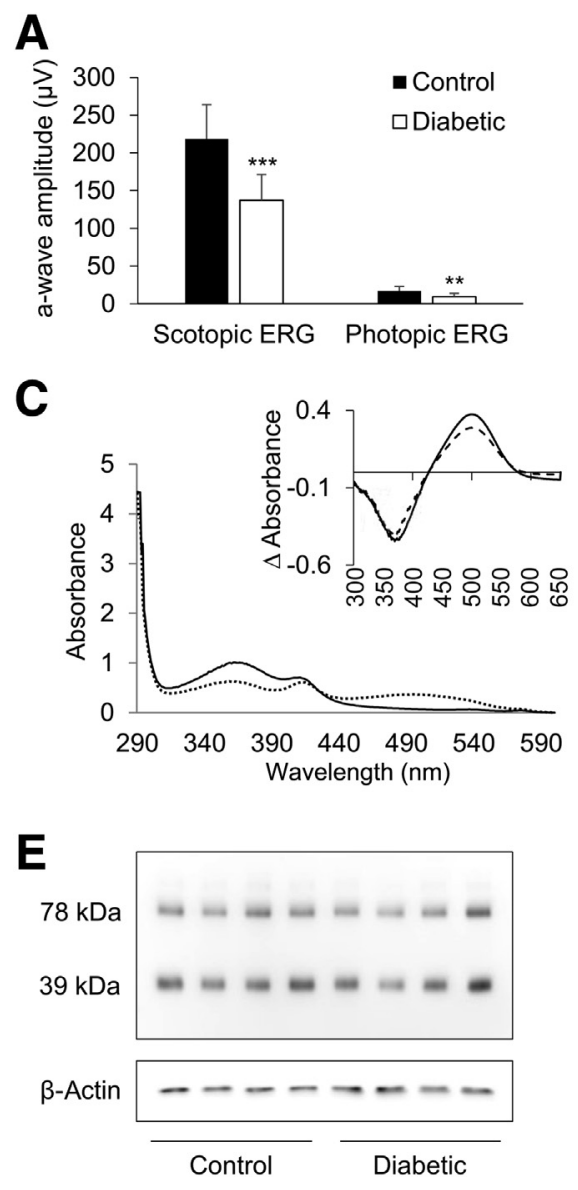

B

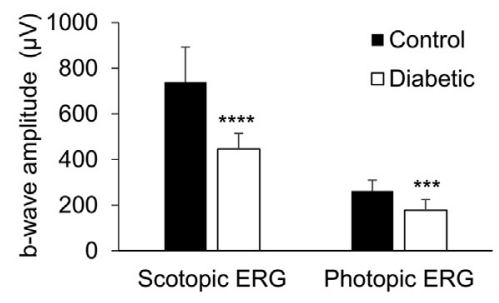

D

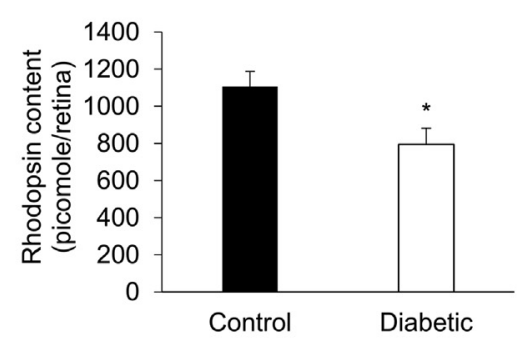

$F$

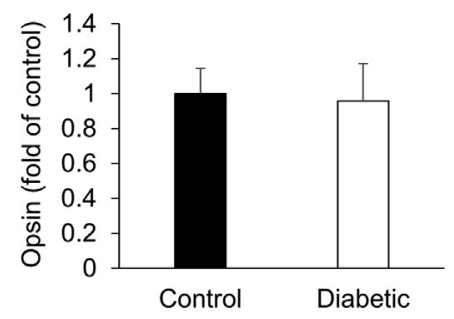

Figure 2 Analysis of retinal function and rhodopsin content changes in diabetes. Retinal function was evaluated in control and diabetic rats at 4 months after diabetes induction by electroretinography (ERG). A and $\mathbf{B}$ : The a-wave $(\mathbf{A})$ and the b-wave (B) amplitudes of scotopic and photopic ERG responses from control and diabetic rats are shown on the graph. C: To determine the maximal rhodopsin content in each eye, rats were dark adapted overnight. Rhodopsin levels in control and diabetic rats were measured by absorption spectrophotometry. An absorption spectrum of rhodopsin shows a broad visible absorbance with a maximum wavelength value of $500 \mathrm{~nm}$. The absorption spectrum before rhodopsin bleaching is indicated by the dashed line; the absorption spectrum after rhodopsin bleaching is shown by the solid line. Inset: Photobleaching difference spectrum obtained by subtracting the light spectrum from the dark spectrum in control (solid line) and diabetic (dashed line) retinas. D: Rhodopsin content in each eye was calculated as picomoles/ retina. E: Expression levels of opsin in the retinas of control and diabetic rats were measured by Western blot using an anti-rhodopsin (1D4) antibody. F: Opsin monomer level was analyzed by densitometry and normalized by $\beta$-actin level. Data are expressed as means \pm SD (A, B, D, and $\mathbf{F})$. $N=8(\mathbf{A}$ and $\mathbf{B}) ; N=13(\mathbf{D}) ; N=4(\mathbf{F})$. ${ }^{*} P<0.05,{ }^{* *} P<0.01,{ }^{* * *} P<0.001$, and $* * * * P<0.0001$. thick) by cryostat microtome Leica CM3050 S (Leica Biosystems Inc., Buffalo Grove, IL). Thereafter, cross sections were stained with an antibody specific for STRA6 (Abcam).

\section{Statistical Analysis}

Quantitative data are expressed as means $\pm \mathrm{SD}$. A $t$-test was used to assess differences between two groups. In all cases, $P<0.05$ was considered as statistically significant.

\section{Results}

Average blood glucose concentrations and body weights of both diabetic and nondiabetic control rats at the beginning of experiments and by the end of the 4-month study are summarized in Supplemental Figures S1 and S2. Diabetic rats had reduced body weights compared to control rats $(P<0.0001)$ and higher blood glucose levels $(P<0.0001)$ throughout the 4-month study.

\section{Photoreceptor Function in Diabetic Rats Is Impaired at 4 Months after Induction of Diabetes}

Electrophysiological analysis was performed in eight diabetic and eight nondiabetic control rats. ERG responses were recorded at 4 months after induction of diabetes. The maximal amplitudes of both scotopic and photopic a- and bwaves were significantly lower in diabetic rats compared to nondiabetic control rats (Figure 2, A and B, and Supplemental Figure S3), suggesting an impaired visual function in diabetic rats.

\section{Rhodopsin Is Decreased in the Retina of Diabetic Rats}

It is well known that mutations or deletion of genes involved in the visual cycle decreases or completely diminishes ERG response. ${ }^{16,17}$ Therefore, we hypothesize that decreased responses of ERG in diabetic rats might be because of disturbed regeneration of visual pigments. To test this hypothesis, we analyzed rhodopsin levels in diabetic rats at 4 months after the onset of diabetes and in their age-matched nondiabetic control rats. Total rhodopsin was extracted with detergent from darkadapted retinas. The rhodopsin absorption maximum was observed at $500 \mathrm{~nm}$ (Figure 2C). The difference of absorbance spectra between unbleached and bleached visual pigments was measured to quantify endogenous rhodopsin. The amount of total rhodopsin was 30\% lower in diabetic rat retinas, compared to that in the control group (Figure 2D). To determine whether decrease of rhodopsin levels in diabetic animals might be a consequence of the reduction of opsin expression or 

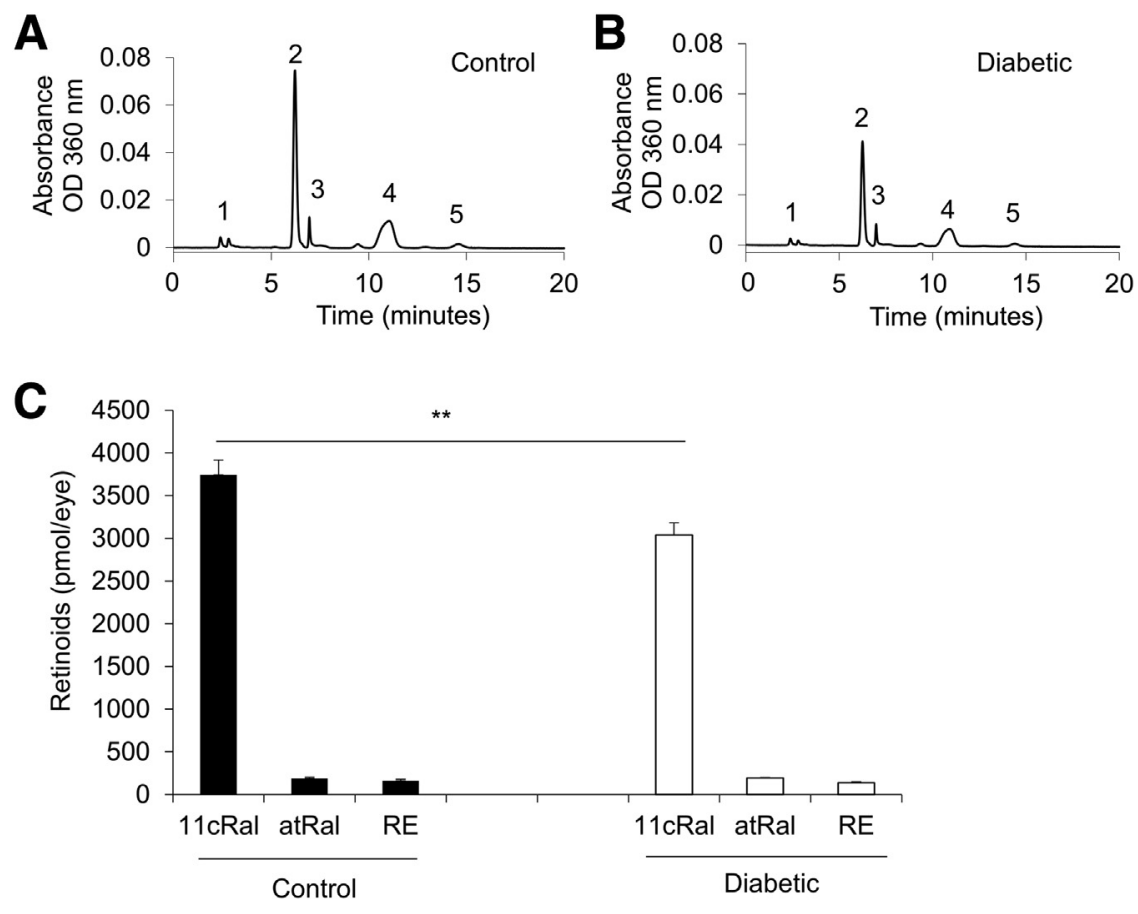

Figure 3 Endogenous retinoid profiles of darkadapted diabetic and control rat eyecups. At 4 months of age, endogenous retinoids were extracted from the rat eyecups and analyzed by high-performance liquid chromatography (HPLC). A and B: Representative HPLC chromatograms at $360 \mathrm{~nm}$ are shown for control (A) and diabetic (B) rats. Peaks were identified according to retinoid standards as follows: 1 , retinyl esters; 2 , syn-11cis-retinal oxime; 3, syn-all-trans-retinal oxime; 4, anti-11-cis-retinal-oxime; 5, anti-all-trans-retinal oxime. C: Amounts of retinoids, such as 11-cisretinal (11cRal), all-trans-retinal (atRal), and retinyl esters (REs), were quantified by measuring the peak areas and averaged within the group. Data are expressed as means \pm SD (C). $N=8$ (C). ${ }^{* *} P<0.01 .0 \mathrm{D}$, optical density. the decrease in 11-cis-retinal chromophore, first we analyzed opsin expression levels in control and diabetic retinas by Western blotting, which showed characteristic presence of dimeric forms of opsin. As shown by Western blot analysis, opsin levels were similar in both groups (Figure 2, E and F), suggesting that diabetes did not affect opsin expression or stability at that time point.

\section{1-cis-Retinal Levels Are Reduced in Eyes of Diabetic Rats}

To determine the cause of rhodopsin decrease in diabetic retina, we measured 11-cis-retinal chromophore as well as alltrans-retinal and retinyl ester concentrations in the eyes of overnight dark-adapted control and diabetic animals at 4 months after the onset of diabetes by HPLC. Retinoids were extracted in the presence of hydroxylamine to disrupt the Schiff bond and convert retinoids to corresponding retinal oximes. The amount of 11-cis-retinal per eye was decreased by approximately $30 \%$ in diabetic rat eyes as compared to that in nondiabetic controls (Figure 3), suggesting that generation of chromophore is deficient in diabetic rats, and that a significant portion of opsin remains unbound with 11-cis-retinal. The levels of all-trans-retinal and retinyl esters were insignificantly different in both groups. To determine whether the decrease in 11-cis-retinal generation is ascribed to a deficient visual cycle, we analyzed expression levels of visual cycle proteins. As shown by Western blot analysis, no significant difference was found in expression levels of LRAT and RPE65 in the RPE of diabetic versus control rats at the same time point as for retinoid analysis (Figure 4, A-D). Enzymatic activities of LRAT and RPE65 were also similar in diabetic and control rats
(Figure 4, E and F). In contrast, levels of STRA6 and IRBP were significantly down-regulated in diabetic eyes compared to nondiabetic controls (Figure 5, A-C). Immunohistochemistry analysis of eyecup cross sections has confirmed reduced STRA6 expression in the RPE of diabetic rats compared to nondiabetic animals, despite the normal retinal structure in both diabetic and control groups (Figure 5D).

\section{RBP4 and Retinyl Ester Levels Are Lower in Serum of Diabetic Rats}

Retinol is delivered to the RPE by RBP4 via interaction with STRA6 on its basolateral plasma membrane. Because RBP4 is the major carrier of retinol in the blood, and its abundancy may affect retinoid supply to the eye, we measured RBP4 levels in serum of diabetic and control rats using enzymelinked immunosorbent assay. We also measured all-transretinol and retinyl ester concentrations in serum of diabetic and control animals using HPLC. Both serum levels of RBP4 and retinyl ester were lower in diabetic rats at 4 months after the onset of diabetes, compared to those of the control rats (Figure 6). However, the difference in all-transretinol levels was not statistically significant between the two groups. The decrease in levels of plasma retinoids and retinol carrier may lead to an insufficient vitamin A supply to retinoid-dependent tissues and organs, such as the eye.

\section{Discussion}

DR remains a leading cause of severe vision loss and blindness. ${ }^{1,2}$ Recently, accumulating evidence suggests that 
A

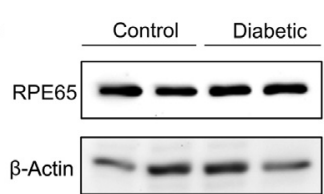

C

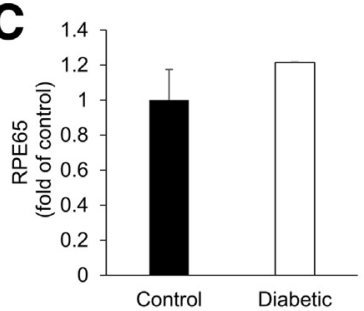

$\mathbf{E}$

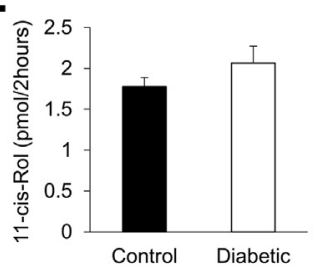

B

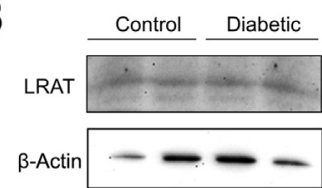

D $\quad 1.4$

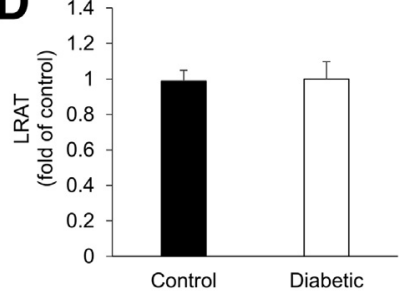

$\mathbf{F}$

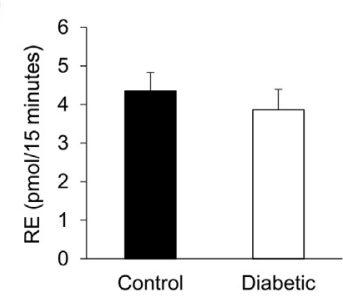

Figure 4 Protein levels and enzymatic activities of retinal pigment epithelium-specific 65-kDa protein (RPE65) and lecithin retinol acyltransferase (LRAT) in diabetic and control rats. The protein levels of RPE65 and LRAT were measured in the eyecups of control and diabetic rats at 4 months after diabetes induction by Western blot analysis using an antiRPE65 (A) and an anti-LRAT (B) antibody. RPE65 (C) and LRAT (D) protein levels were quantified by densitometry and normalized by $\beta$-actin levels. RPE65 (E) and LRAT (F) activities were measured in control and diabetic rat eyecups. Enzymatic activities of RPE65 and LRAT proteins were assayed using high-performance liquid chromatography, as described in Materials and Methods. Data are expressed as means \pm SD $(\mathbf{C}-\mathbf{F}) . N=4$ (control and diabetic rats) (C-F).

DR is not simply a retinal vascular disease. The functions of the neural retina are also impaired in early diabetes before the structural changes in the retina and vasculature. ${ }^{18,19}$ However, the pathogenic mechanism affecting retinal function in early stages of DR has not been well investigated. The present study provided the first evidence demonstrating that the retinoid metabolism is disturbed in diabetes, leading to deficient generation of 11-cis-retinal, the chromophore essential for visual pigment formation. This is supported by the reduced rhodopsin levels, whereas opsin expression remained unchanged in the diabetic retina. The deficient generation of chromophore and visual pigments may contribute to visual function defects, such as delayed dark adaptation in early DR, a new pathogenic mechanism of DR. Chronic deficiency in visual pigment formation may contribute to photoreceptor degeneration and irreversible retinal pathologies in advanced DR. This study suggests that restoration of the normal retinoid level may represent a potential therapeutic strategy for early DR.

In line with previous studies, ${ }^{18,20}$ ERG recording demonstrated significantly reduced a- and b-wave amplitudes in STZ-induced diabetic animals, suggesting photoreceptor dysfunction. We believe that a $30 \%$ reduction of

chromophore, as demonstrated by HPLC and rhodopsin assay, translates to functional deficits across all intensities, and that a greater reduction at a low light sensitivity in diabetic groups may be attributed to mild retinal degeneration. Our results are also consistent with the previously reported data for vitamin A-deprived rats, albeit the difference in our diabetic rat model was less severe. ${ }^{21} \mathrm{We}$ hypothesized that decreased electrophysiological response might be ascribed to impaired visual pigment generation. Indeed, previously it has been reported that rhodopsin regeneration was decreased in excised diabetic mouse eyes. ${ }^{22}$ However, those data were obtained for an ex vivo eye perfused with glucose; therefore, they may not be directly related to in vivo diabetic conditions. Herein, we measured rhodopsin levels in dark-adapted diabetic and control animals and found that rhodopsin levels are significantly decreased after 4 months of STZ-induced diabetes. As rhodopsin consists of opsin and the chromophore, 11-cis-retinal, decreases in rhodopsin levels may be ascribed to either down-regulated opsin expression or the diminished 11-cis-retinal chromophore, which is regenerated through the visual cycle. To determine whether opsin expression level is changed during diabetes, we measured opsin levels

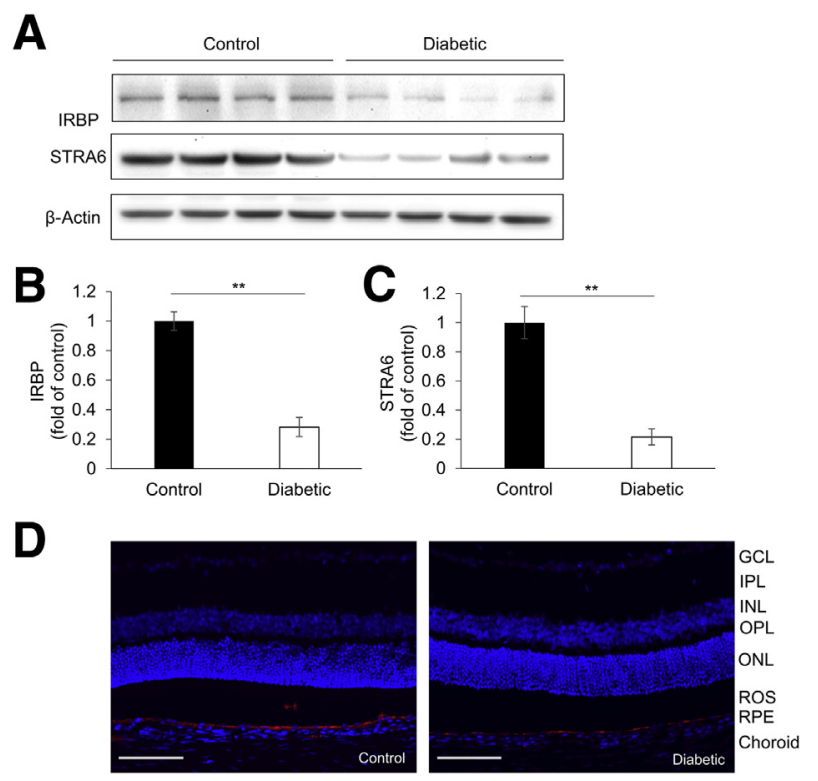

Figure 5 Protein levels of interphotoreceptor retinoid-binding protein (IRBP) and stimulated by retinoic acid 6 (STRA6) in diabetic and control rats. A: The protein levels of IRBP and STRA6 were measured in the eyecups of diabetic and control rats at 4 months after diabetes induction by Western blot using an anti-IRBP and an anti-STRA6 antibody. B and C: IRBP (B) and STRA6 (C) protein levels were quantified by densitometry and normalized by $\beta$-actin levels. D: STRA6 expression is down-regulated in the retinal pigment epithelium (RPE) of diabetic rats. Eyecup tissue cross sections from control and diabetic rats were stained with an antibody specific for STRA6 (red) by immunohistochemistry. The nuclei were counterstained with DAPI (blue). Processing time was equal among experimental groups. Data are expressed as means \pm SD (B and C). $N=4$ (B and C). ${ }^{* *} P<0.01$. Scale bar $=100 \mu \mathrm{m}$ (D). GCL, ganglion cell layer; INL, inner nuclear layer; IPL, inner plexiform layer; ONL, outer nuclear layer; $\mathrm{OPL}$, outer plexiform layer; ROS, receptor outer segment. 

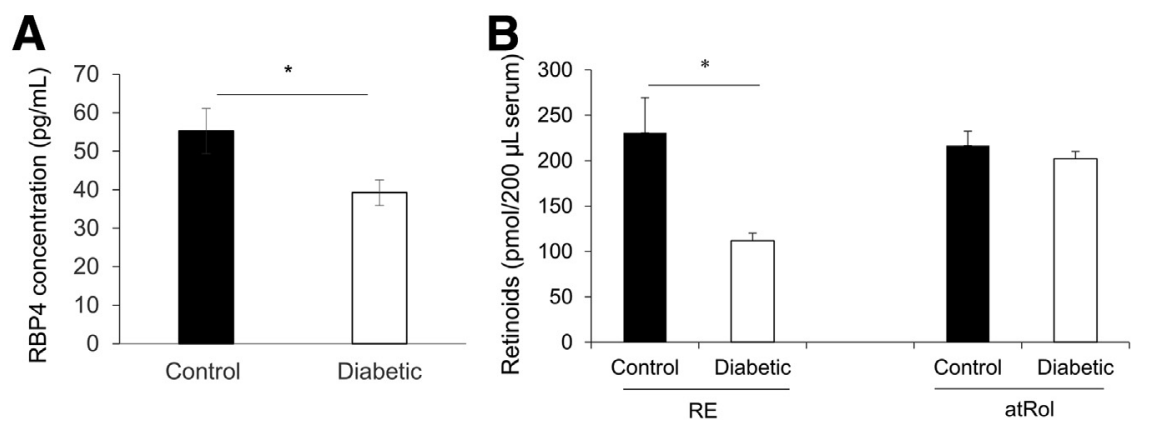

Figure 6 Retinol-binding protein 4 (RBP4) and retinoid concentrations in rat serum. A: RBP4 serum concentrations in control and diabetic rats were measured by enzyme-linked immunosorbent assay. B: Serum concentrations of retinyl esters (REs) and all-trans-retinol (atRol) in control and diabetic rats were determined by highperformance liquid chromatography. Data are expressed as means \pm SD. $N=7$ (A); $N=6$ (B). $* P<0.05$. using Western blot analysis. Our results showed that opsin expression in diabetic rats was similar to that in nondiabetic control retinas, which is in line with the previous observation that opsin mRNA levels are unchanged in rats after 4 weeks of diabetes. $^{23}$

Another possible cause of the decreased rhodopsin concentration in diabetic rats might be the reduced generation of 11-cis-retinal chromophore. Indeed, we found that the 11-cis-retinal level was $30 \%$ lower in diabetic rats, which corresponds to the same degree of reduction in rhodopsin level. Interestingly, reduced 11-cis-retinal concentrations in diabetic retinas were observed previously for diabetic rats $^{24}$; however, the absolute values of 11-cisretinal reported in that study were extremely low (only 52 pmol per retina for nondiabetic rats). Most likely, this could be explained by a different method of retinoid extraction from the retina used by the authors, who did not use hydroxylamine to disrupt the Schiff base bond between 11-cis-retinal and opsin; thus, the extraction of retinal might have been incomplete. Decreased levels of 11-cisretinal might be a result of the impaired visual cycle. RPE65 catalyzes the rate-limiting step of the retinoid cycle and produces 11-cis-retinol, which is a direct precursor of 11-cis-retinal. ${ }^{25}$ To our surprise, our results did not show any significant difference in expression levels of LRAT and RPE65, which are the key enzymes of the visual cycle. Previously, another group showed by immunohistochemistry that the RPE65 expression level is lower in the RPE of diabetic rats after 3 months of diabetes; however, the authors did not present quantitative comparison of RPE65 protein levels. ${ }^{23}$ Moreover, according to their own data, RPE65 mRNA levels in the RPE were similar in diabetic and control rats.

We did not detect a significant difference in retinyl ester levels between dark-adapted diabetic and nondiabetic animals. Retinyl ester is stored in retinosomes, and its levels depend on relative activities of LRAT and yet to be identified retinyl ester hydrolase. Previously, it has been shown that retinyl ester hydrolase is depressed in the liver of diabetic rats. ${ }^{26} \mathrm{~A}$ similar phenomenon may take place in the RPE. Therefore, even though total retinol flow is decreased in the diabetic eye, the retinyl ester level may remain unchanged in the diabetic RPE.
The binding of 11-cis-retinal to bleached visual pigment prevents the activation of the phototransduction cascade by a pool of accumulated apo-opsin. It has been reported that increased levels of chromophore-free opsin, as found in animals with mutations of RPE65 and $L R A T^{27}$ or vitamin A deficiency, ${ }^{28}$ have deleterious effects on photoreceptor cells. Chromophore-free opsin is known to constitutively activate the phototransduction pathway. ${ }^{29,30}$ Constitutive activity of free opsin desensitizes photoreceptor cells and causes retinal degeneration. ${ }^{31}$ In early diabetes, because of decreased 11cis-retinal generation, rhodopsin levels are reduced, whereas retinal morphology and opsin expression remain unchanged, resulting in increased amount of unsaturated opsin. The excess amount of free opsin may contribute to photoreceptor dysfunction in advanced DR.

IRBP (RBP3) is an essential protein of the visual cycle present in the interphotoreceptor space, which binds 11-cisretinal and all-trans-retinol and facilitates their transport between the RPE and photoreceptors. ${ }^{32}$ We found that IRBP was down-regulated in diabetic rats. In a human study, it has been reported that the expression level of IRBP is also decreased in patients with DR at early stages. ${ }^{33,34}$ IRBP protects 11-cis-retinal from photodegradation and photoisomerization. ${ }^{35,36}$ There is some evidence that IRBP also promotes retinol uptake and release by rat Müller cells. ${ }^{37}$ $I R B P^{-I-}$ mice displayed decreased ERG amplitudes ${ }^{38-40}$ and photoreceptor degeneration at postnatal day $25 .^{41}$ Therefore, it is likely that decreased IRBP levels contribute to reduced 11-cis-retinal levels in diabetic rats.

Although most of 11-cis-retinal is regenerated through the visual cycle, a part of it is irreversibly degraded because of photo-oxidation. ${ }^{42}$ Therefore, a constant supply of retinol from the blood circulation is necessary to maintain sufficient retinoid levels in the eye. Currently, it is established that efficient delivery of vitamin A to the eye is mediated through RBP4 and its receptor STRA6. RBP4 plays a key role in mobilization of retinol from the liver and transporting it to the eye and other organs. STRA6 facilitates flux of retinol between RBP4 in the circulation and the RPE. Both $R_{B P 4^{-1-}}$ and STRA6 ${ }^{-1-}$ mice have decreased ERG amplitudes of $\mathrm{a}$ and $\mathrm{b}$ waves and decreased amounts of retinoids in the retina. ${ }^{43-45} \mathrm{RBP} 4$ levels in serum of STZ-diabetic rats were significantly reduced, as shown by enzyme-linked 
immunosorbent assay in the current study, consistent with previous reports by other groups. ${ }^{24,45}$ Although serum retinol levels were not significantly lower in diabetic rats in our study, retinyl ester concentrations were found to be lower in diabetic rats compared to those of control. These results are in agreement with previous studies, including studies of type 1 diabetes patients ${ }^{47}$ and STZ-induced diabetic rats. ${ }^{24,48}$ Several other human studies have also reported that in patients with type 1 diabetes, plasma levels of retinol and RBP4 are decreased. ${ }^{49}$

In diabetes, the mechanism of vitamin A deficiency in serum is unclear. It has been shown that patients with type 1 diabetes have elevated carotenoid and retinyl ester levels, which suggests that their conversion to retinol is decreased. ${ }^{50}$ The expression of $\beta$-carotene monooxygenase was shown to be reduced in diabetic rats. ${ }^{46} \beta$-Carotene monooxygenase has a critical role in the metabolism of $\beta$-carotene to retinal; therefore, the decreased $\beta$-carotene monooxygenase expression might change the serum retinol level. Nevertheless, it is known that diabetic rats have an increased storage of retinyl esters in the liver as a result of excessive consumption of food under insulin-deficient conditions. It has been suggested that mobilization of retinol from the liver, which is regulated by unidentified retinyl ester hydrolase, is decreased in diabetic rats. ${ }^{51}$ Indeed, the hepatic retinyl ester hydrolase activity was depressed at the onset of diabetes in biobreeding rats. ${ }^{26}$ RBP4 circulates as a complex with transthyretin, a homotetrameric thyroxine transport protein. ${ }^{52}$ The declined level of retinoids and RBP4 in blood circulation might be also a result of increased renal filtration because of lower plasma concentrations of transthyretin in type 1 diabetes. ${ }^{49}$

The present study also demonstrated, for the first time, the decrease in STRA6 levels in the RPE of diabetic rats. $S T R A 6^{-1-}$ mice displayed drastically decreased contents of retinoids in the RPE and retina, suggesting that STRA6 is critical for retinoid uptake in the visual cycle. ${ }^{43}$ It is likely that the decrease of serum levels of RBP4 and retinol, together with decrease of the STRA6 receptor, results in a significant local vitamin A deficiency in the eye, which may subsequently lead to a decreased rhodopsin level in diabetic rats. Previously, we have demonstrated that the mice maintained on a vitamin A-deficient diet have a significantly decreased endogenous 11-cis-retinal level in the eye. ${ }^{53} \mathrm{We}$ speculate that local vitamin A deficiency in the eye might be corrected by the additional vitamin $\mathrm{A}$ in the diet. Alternatively, the 11or 9-cis-retinal chromophore may be delivered to serum of diabetic patients to ameliorate photoreceptor dysfunction. This notion is supported by a recent study showing that the administration of 11- and 9-cis-retinal to diabetic mice corrected visual abnormalities induced by diabetes, as shown by maximal rod response and optokinetic tracking. ${ }^{54}$

Paradoxically, it has been proposed that the inhibition of the visual cycle may ameliorate the pathogenesis of lesions characteristic of early DR in diabetic mice. ${ }^{55}$ The authors used a retinoid, retinylamine, to inhibit the visual cycle in their study. However, because retinylamine serves as a substrate for LRAT, it may be accumulated in the RPE and metabolized to other retinoids, including retinoic acid, which binds to retinoic acid receptors and regulates expression of multiple genes. ${ }^{56,57}$ Thus, the results of this study may not be solely ascribed to the inhibition of the visual cycle. Moreover, it has been recently shown that both retinylamine and emixustat (another inhibitor of the visual cycle, produced by Acucela, Seattle, WA) most likely exert their effects not through the inhibition of the visual cycle, but rather because of sequestration of toxic all-trans-retinal via the Schiff base acting as a scavenger. ${ }^{58}$

In summary, our results have provided the first evidence that rhodopsin levels are decreased and 11-cis-retinal generation is impaired in diabetic eyes. Most likely, disturbed rhodopsin generation is a result of the down-regulation of IRBP, STRA6, RBP4, and, consequently, relative vitamin A deficiency in ocular tissues in diabetes. Although the mechanism by which diabetes affects serum vitamin A levels and the expression levels of IRBP, RBP4, and STRA6 remains to be elucidated, this study reveals a new aspect of DR and demonstrates a previously unknown association between the retinoid metabolism and diabetes. These findings may lead to new understanding of the retinal dysfunction mechanism in early DR and contribute to the development of a new therapeutic strategy for DR.

\section{Acknowledgments}

V.V.M. performed research, analyzed data, and wrote the manuscript; Y.T. and Y.S. performed experiments; J.-x.M. designed the study, participated in data analysis, and wrote the manuscript; G.M. designed the study, performed experiments, participated in data analysis, and wrote the manuscript; and G.M. is the guarantor of this work and, as such, had full access to all of the data in the study and takes responsibility for the integrity of the data and the accuracy of the data analysis.

\section{Supplemental Data}

Supplemental material for this article can be found at doi:http://dx.doi.org/10.1016/j.ajpath.2017.06.007.

\section{References}

1. Klein BE: Overview of epidemiologic studies of diabetic retinopathy. Ophthalmic Epidemiol 2007, 14:179-183

2. Congdon NG, Friedman DS, Lietman T: Important causes of visual impairment in the world today. JAMA 2003, 290:2057-2060

3. Crawford TN, Alfaro DV 3rd, Kerrison JB, Jablon EP: Diabetic retinopathy and angiogenesis. Curr Diabetes Rev 2009, 5:8-13

4. Daley ML, Watzke RC, Riddle MC: Early loss of blue-sensitive color vision in patients with type I diabetes. Diabetes Care 1987, 10: 777-781

5. Henson DB, North RV: Dark adaptation in diabetes mellitus. Br J Ophthalmol 1979, 63:539-541 
6. Lieth E, Gardner TW, Barber AJ, Antonetti DA; Penn State Retina Research Group: Retinal neurodegeneration: early pathology in diabetes. Clin Exp Ophthalmol 2000, 28:3-8

7. Wald G: Molecular basis of visual excitation. Science 1968, 162: 230-239

8. Kiser PD, Golczak M, Palczewski K: Chemistry of the retinoid (visual) cycle. Chem Rev 2014, 114:194-232

9. Kawaguchi R, Yu J, Honda J, Hu J, Whitelegge J, Ping P, Wiita P, Bok D, Sun H: A membrane receptor for retinol binding protein mediates cellular uptake of vitamin A. Science 2007, 315:820-825

10. Amengual J, Zhang N, Kemerer M, Maeda T, Palczewski K, Von Lintig J: STRA6 is critical for cellular vitamin A uptake and homeostasis. Hum Mol Genet 2014, 23:5402-5417

11. Gonzalez-Fernandez F: Interphotoreceptor retinoid-binding protein: an old gene for new eyes. J Ophthalmic Vis Res 2003, 43:3021-3036

12. Wu Q, Blakeley LR, Cornwall MC, Crouch RK, Wiggert BN, Koutalos Y: Interphotoreceptor retinoid-binding protein is the physiologically relevant carrier that removes retinol from rod photoreceptor outer segments. Biochemistry 2007, 46:8669-8679

13. Irreverre F, Stone AL, Shichi H, Lewis MS: Biochemistry of visual pigments, I: purification and properties of bovine rhodopsin. J Biol Chem 1969, 244:529-536

14. Bradford MM: A rapid and sensitive method for the quantitation of microgram quantities of protein utilizing the principle of protein-dye binding. Anal Biochem 1976, 72:248-254

15. Moiseyev G, Crouch RK, Goletz P, Oatis J Jr, Redmond TM, Ma JX: Retinyl esters are the substrate for isomerohydrolase. Biochemistry 2003, 42:2229-2238

16. Redmond TM, Yu S, Lee E, Bok D, Hamasaki D, Chen N, Goletz P, Ma JX, Crouch RK, Pfeifer K: Rpe65 is necessary for production of 11-cis-vitamin A in the retinal visual cycle. Nat Genet 1998, 20:344-351

17. Ruiz A, Ghyselinck NB, Mata N, Nusinowitz S, Lloyd M, Dennefeld C, Chambon P, Bok D: Somatic ablation of the Lrat gene in the mouse retinal pigment epithelium drastically reduces its retinoid storage. Invest Ophthalmol Vis Sci 2007, 48:5377-5387

18. Aung MH, Kim MK, Olson DE, Thule PM, Pardue MT: Early visual deficits in streptozotocin-induced diabetic long evans rats. Invest Ophthalmol Vis Sci 2013, 54:1370-1377

19. Jackson GR, Scott IU, Quillen DA, Walter LE, Gardner TW: Inner retinal visual dysfunction is a sensitive marker of non-proliferative diabetic retinopathy. Br J Ophthalmol 2012, 96:699-703

20. Phipps JA, Yee P, Fletcher EL, Vingrys AJ: Rod photoreceptor dysfunction in diabetes: activation, deactivation, and dark adaptation. Invest Ophthalmol Vis Sci 2006, 47:3187-3194

21. Katz ML, Chen DM, Stientjes HJ, Stark WS: Photoreceptor recovery in retinoid-deprived rats after vitamin A replenishment. Exp Eye Res 1993, 56:671-682

22. Ostroy SE, Frede SM, Wagner EF, Gaitatzes CG, Janle EM: Decreased rhodopsin regeneration in diabetic mouse eyes. Invest Ophthalmol Vis Sci 1994, 35:3905-3909

23. Kirwin SJ, Kanaly ST, Hansen CR, Cairns BJ, Ren M, Edelman JL: Retinal gene expression and visually evoked behavior in diabetic long evans rats. Invest Ophthalmol Vis Sci 2011, 52:7654-7663

24. Tuitoek PJ, Ziari S, Tsin AT, Rajotte RV, Suh M, Basu TK: Streptozotocin-induced diabetes in rats is associated with impaired metabolic availability of vitamin A (retinol). Br J Nutr 1996, 75:615-622

25. Moiseyev G, Chen Y, Takahashi Y, Wu BX, Ma JX: RPE65 is the isomerohydrolase in the retinoid visual cycle. Proc Natl Acad Sci U S A $2005,102: 12413-12418$

26. Chen M, Thomson AB, Tsin AT, Basu TK: The hepatic retinyl ester hydrolase activity is depressed at the onset of diabetes in $\mathrm{BB}$ rats. $\mathrm{Br} \mathrm{J}$ Nutr 2003, 89:231-238

27. Fan J, Rohrer B, Frederick JM, Baehr W, Crouch RK: Rpe65-/- and Lrat-/- mice: comparable models of leber congenital amaurosis. Invest Ophthalmol Vis Sci 2008, 49:2384-2389

28. Dowling JE, Wald G: Vitamin A deficiency and night blindness. Proc Natl Acad Sci U S A 1958, 44:648-661
29. Cornwall MC, Fain GL: Bleached pigment activates transduction in isolated rods of the salamander retina. J Physiol 1994, 480:261-279

30. Fan J, Woodruff ML, Cilluffo MC, Crouch RK, Fain GL: Opsin activation of transduction in the rods of dark-reared Rpe65 knockout mice. J Physiol 2005, 568:83-95

31. Woodruff ML, Wang Z, Chung HY, Redmond TM, Fain GL, Lem J: Spontaneous activity of opsin apoprotein is a cause of Leber congenital amaurosis. Nat Genet 2003, 35:158-164

32. Crouch RK, Hazard ES, Lind T, Wiggert B, Chader G, Corson DW: Interphotoreceptor retinoid-binding protein and alpha-tocopherol preserve the isomeric and oxidation state of retinol. Photochem Photobiol 1992, 56:251-255

33. Garcia-Ramirez M, Hernandez C, Villarroel M, Canals F, Alonso MA, Fortuny R, Masmiquel L, Navarro A, Garcia-Arumi J, Simo R: Interphotoreceptor retinoid-binding protein (IRBP) is downregulated at early stages of diabetic retinopathy. Diabetologia 2009, 52:2633-2641

34. Garcia-Ramirez M, Canals F, Hernandez C, Colome N, Ferrer C, Carrasco E, Garcia-Arumi J, Simo R: Proteomic analysis of human vitreous fluid by fluorescence-based difference gel electrophoresis (DIGE): a new strategy for identifying potential candidates in the pathogenesis of proliferative diabetic retinopathy. Diabetologia 2007, 50:1294-1303

35. Parker R, Wang JS, Kefalov VJ, Crouch RK: Interphotoreceptor retinoid-binding protein as the physiologically relevant carrier of 11-cis-retinol in the cone visual cycle. J Neurosci 2011, 31: 4714-4719

36. Gonzalez-Fernandez F, Betts-Obregon B, Yust B, Mimun J, Sung D, Sardar D, Tsin AT: Interphotoreceptor retinoid-binding protein protects retinoids from photodegradation. Photochem Photobiol 2015, 91:371-378

37. Betts-Obregon BS, Gonzalez-Fernandez F, Tsin AT: Interphotoreceptor retinoid-binding protein (IRBP) promotes retinol uptake and release by rat Müller cells (rMC-1) in vitro: implications for the cone visual cycle. Invest Ophthalmol Vis Sci 2014, 55:6265-6271

38. Liou GI, Fei Y, Peachey NS, Matragoon S, Wei S, Blaner WS, Wang Y, Liu C, Gottesman ME, Ripps H: Early onset photoreceptor abnormalities induced by targeted disruption of the interphotoreceptor retinoid-binding protein gene. J Neurosci 1998, 18:4511-4520

39. Parker RO, Fan J, Nickerson JM, Liou GI, Crouch RK: Normal cone function requires the interphotoreceptor retinoid binding protein. J Neurosci 2009, 29:4616-4621

40. Jin M, Li S, Nusinowitz S, Lloyd M, Hu J, Radu RA, Bok D, Travis GH: The role of interphotoreceptor retinoid-binding protein on the translocation of visual retinoids and function of cone photoreceptors. J Neurosci 2009, 29:1486-1495

41. Wisard J, Faulkner A, Chrenek MA, Waxweiler T, Waxweiler W, Donmoyer C, Liou GI, Craft CM, Schmid GF, Boatright JH, Pardue MT, Nickerson JM: Exaggerated eye growth in IRBPdeficient mice in early development. Invest Ophthalmol Vis Sci 2011, 52:5804-5811

42. Ueda K, Zhao J, Kim HJ, Sparrow JR: Photodegradation of retinal bisretinoids in mouse models and implications for macular degeneration. Proc Natl Acad Sci U S A 2016, 113:6904-6909

43. Ruiz A, Mark M, Jacobs H, Klopfenstein M, Hu J, Lloyd M, Habib S, Tosha C, Radu RA, Ghyselinck NB, Nusinowitz S, Bok D: Retinoid content, visual responses, and ocular morphology are compromised in the retinas of mice lacking the retinol-binding protein receptor, STRA6. Invest Ophthalmol Vis Sci 2012, 53:3027-3039

44. Quadro L, Blaner WS, Salchow DJ, Vogel S, Piantedosi R, Gouras P, Freeman S, Cosma MP, Colantuoni V, Gottesman ME: Impaired retinal function and vitamin A availability in mice lacking retinolbinding protein. EMBO J 1999, 18:4633-4644

45. Vogel S, Piantedosi R, O’Byrne SM, Kako Y, Quadro L, Gottesman ME, Goldberg IJ, Blaner WS: Retinol-binding proteindeficient mice: biochemical basis for impaired vision. Biochemistry 2002, 41:15360-15368 
46. Takitani K, Inoue K, Koh M, Miyazaki H, Inoue A, Kishi K, Tamai H: Altered retinol status and expression of retinol-related proteins in streptozotocin-induced type 1 diabetic model rats. J Clin Biochem Nutr 2015, 56:195-200

47. Basu TK, Tze WJ, Leichter J: Serum vitamin A and retinol-binding protein in patients with insulin-dependent diabetes mellitus. Am J Clin Nutr 1989, 50:329-331

48. Tuitoek PJ, Ritter SJ, Smith JE, Basu TK: Streptozotocin-induced diabetes lowers retinol-binding protein and transthyretin concentrations in rats. Br J Nutr 1996, 76:891-897

49. Pullakhandam R, Palika R, Ghosh S, Reddy GB: Contrasting effects of type 2 and type 1 diabetes on plasma RBP4 levels: the significance of transthyretin. IUBMB Life 2012, 64:975-982

50. Granado F, Olmedilla B, Gil-Martinez E, Blanco I, Millan I, RojasHidalgo E: Carotenoids, retinol and tocopherols in patients with insulin-dependent diabetes mellitus and their immediate relatives. Clin Sci 1998, 94:189-195

51. Lu J, Dixon WT, Tsin AT, Basu TK: The metabolic availability of vitamin $\mathrm{A}$ is decreased at the onset of diabetes in BB rats. J Nutr 2000, 130:1958-1962

52. D'Ambrosio DN, Clugston RD, Blaner WS: Vitamin A metabolism: an update. Nutrients 2011, 3:63-103
53. Hu Y, Chen Y, Moiseyev G, Takahashi Y, Mott R, Ma JX: Comparison of ocular pathologies in vitamin A-deficient mice and RPE65 gene knockout mice. Invest Ophthalmol Vis Sci 2011, 52:5507-5514

54. Berkowitz BA, Kern TS, Bissig D, Patel P, Bhatia A, Kefalov VJ, Roberts R: Systemic retinaldehyde treatment corrects retinal oxidative stress, rod dysfunction, and impaired visual performance in diabetic mice. Invest Ophthalmol Vis Sci 2015, 56:6294-6303

55. Liu H, Tang J, Du Y, Lee CA, Golczak M, Muthusamy A, Antonetti DA, Veenstra AA, Amenqual J, von Lintig J, Palczewski K, Kern TS: Retinylamine benefits early diabetic retinopathy in mice. J Biol Chem 2015, 290:21568-21579

56. Mitchell DM, Stevens CB, Frey RA, Hunter SS, Ashino R, Kawamura S, Stenkamp DL: Retinoic acid signaling regulates differential expression of the tandemly-duplicated long wavelengthsensitive cone opsin genes in zebrafish. PLoS Genet 2015, 11: e1005483

57. Cvekl A, Wang WL: Retinoic acid signaling in mammalian eye development. Exp Eye Res 2009, 89:280-291

58. Zhang J, Kiser PD, Badiee M, Palczewska G, Dong Z, Golczak M, Tochtrop GP, Palczewski K: Molecular pharmacodynamics of emixustat in protection against retinal degeneration. J Clin Invest $2015,125: 2781-2794$ 